\title{
Factors Relevant to Knee Pain among Independent Community-Dwelling Older Adults: A Complete Cross-Sectional Study
}

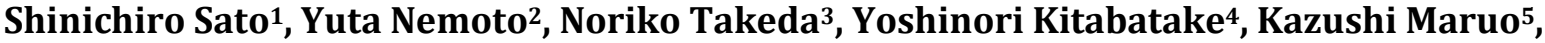 \\ Takashi Arao' \\ ${ }^{1}$ Faculty of Health Sciences, University of Human Arts and Sciences, Saitama, Japan \\ ${ }^{2}$ Research Team for Social Participation and Community Health, Tokyo Metropolitan Institute of Gerontology, Tokyo, Japan \\ ${ }^{3}$ Division of Liberal Arts, Kogakuin University, Tokyo, Japan \\ ${ }^{4}$ Department of Health Sciences, Saitama Prefectural University, Saitama, Japan \\ ${ }^{5}$ Faculty of Medicine, University of Tsukuba, Ibaraki, Japan \\ ${ }^{6}$ Meiji Yasuda Life Foundation of Health and Welfare, Tokyo, Japan \\ Email: shinichiro_sato@human.ac.jp
}

How to cite this paper: Sato, S., Nemoto Y., Takeda, N., Kitabatake, Y., Maruo, K. and Arao, T. (2020) Factors Relevant to Knee Pain among Independent Community-Dwelling Older Adults: A Complete Cross-Sectional Study. Open Journal of Preventive Medicine, 10, 277-287.

https://doi.org/10.4236/ojpm.2020.1011020

Received: September 25, 2020

Accepted: November 15, 2020

Published: November 18, 2020

Copyright $\odot 2020$ by author(s) and Scientific Research Publishing Inc. This work is licensed under the Creative Commons Attribution International License (CC BY 4.0)

http://creativecommons.org/licenses/by/4.0/

\begin{abstract}
Objective: Knee pain is commonly observed among older adults. Therefore, the prevention of knee pain in older adults is a major public health issue. This study aimed to clarify factors relevant to knee pain among older adults. Methods: This study targeted all independent older adults $(\mathrm{N}=6661)$ living in a local municipality in Japan. Participants' health status and lifestyle habits were surveyed using a questionnaire. The survey was conducted from 15 January to 14 February 2016. Relationships between knee pain and physical activity, sitting time, nutrition, drinking, smoking, or body mass index (BMI) were examined using logistic regression analysis. Odds ratios (OR) and $95 \%$ confidence intervals (CI) adjusted for several confounding factors were calculated. Results: Older adults of 5311 responded to the questionnaire and 1843 reported knee pain. The prevalence of knee pain was $34.8 \%$. Knee pain was significantly associated with BMI (OR: 0.60, 95\% CI: $0.51-0.69, P<$ 0.001 in the group of normal range, OR: $0.38,95 \%$ CI: $0.29-0.51, P<0.001$ in the group of $<18.0$ ), nutrition status (OR: $0.63,95 \%$ CI: $0.54-0.75, P<$ 0.001 ), and physical activity (OR: $0.81,95 \%$ CI: $0.71-0.92, P=0.001$ ). Conclusions: This study suggests that one in three independently-living older adults experiences chronic knee pain and that physical activity, BMI, and nutritional status are associated with knee pain.
\end{abstract}

\section{Keywords}

Complete Survey, Physical Activity, Prevalence Rate, Sitting Time 


\section{Introduction}

Musculoskeletal disorders such as rheumatoid arthritis, osteoarthritis (OA), osteoporosis, and severe lower limb injury are commonly observed among older adults, and may be accompanied by chronic pain [1]. Chronic musculoskeletal pain is often observed in the knee joint, and is referred to as "knee pain". Knee pain has a negative impact on physical function, walking ability, subjective health, social participation, and quality of life among older adults [2] [3] [4]. Prevention of knee pain is therefore a major and urgent public health issue for extending healthy life expectancy and decreasing medical and long-term care services costs in aging societies such as Japan and other developed countries [5] [6]. Recent systematic reviews have reported that muscle strengthening and aerobic exercises are effective for improving knee pain [7] [8]. The mechanism of pain relief through exercise is thought to reduce of the burden on the knee joint by muscle strengthening and metabolic effects (e.g., activation of oxidase enzymes and increased capillary blood flow) in response to the exercises. Biomechanical effects such as reduction of the load on the knee joint following weight loss through aerobic exercise are considered to be indirect effects [8] [9]. Based on such evidence, international guidelines for knee pain recommend physical activity as a measure for improving knee pain [9]. Currently, there is insufficient evidence of a primary prevention effect to clarify whether the implementation of physical activity (including the above-mentioned types of exercise) can prevent the onset of knee pain [10]. Various systematic reviews of longitudinal studies on knee pain reported unified factors for the onset of knee pain were obesity, knee trauma, old age, and sex (women). These factors are also reported as risk factors for knee pain in international guidelines [11]. However, no unified views have been established regarding the relationship between knee pain and social factors, health conditions (e.g., medical history and body mass index [BMI]), or lifestyle factors (e.g., physical activity, smoking, Malnutrition, drinking alcohol) [12] [13]. Furthermore, recent research suggests that prolonged sitting time increases the risks for total mortality and coronary mortality; however, the relationship between sedentary behavior and the onset of knee pain (independent from physical activity) has not yet been explored [14] [15]. In previous cohort and cross-sectional studies involving older adults, participants were selected by random or optional sampling and the response rate for questionnaires was about $60 \%$ [16]. Moreover, questionnaire surveys of older adults tend to have a higher rate of missing data than those among younger generations [16]. These findings suggest that in survey-based research involving older adults, a limited number of participants are available for analysis and the results may be influenced by participant selection bias. Therefore, a questionnaire survey study involving older adults should be based on data with low selection bias. This study aimed to clarify the relationship between knee pain and comprehensive factors (including sedentary behavior) based on data with low selection bias drawn from older adults independently living in a community. 


\section{Methods}

\subsection{Participants}

We conducted the survey for every older adult who is 65 years or older and living independently in the municipality with a population of 31,474 people in Japan. Therefore, the selection criteria of the participants were 65 years or older inhabitants of the local municipality and the independency of the daily living. The independent living older adults were defined as the people who were not certified as users of long-term care services by Certification Committee of Needed Long-Term Care in the local government. Therefore, the eligible participants for this study were all independent community-dwelling older adults $(\mathrm{N}=6661)$ selected by excluding the users of long-term care services at the time of the survey from the total number of older adults $(\mathrm{N}=8336)$ living in the municipality.

\subsection{Survey Methods}

The survey was conducted by postal mail using a questionnaire that covered health conditions and lifestyle habits. The survey period was one month (from January 15 to February 14) in 2016. To improve the response rate, a reminder letter was sent to non-respondents one week before the deadline for completed questionnaires. Participants answered the questionnaire and posted it back to the health promotion section in the city hall. The purpose and methods of the survey were printed on the front page of the questionnaire, including a notification informing that those who returned the questionnaire consented to study participation. This study protocol was approved by the ethics committee of Waseda University, (approved number: 2015-218).

\subsection{Measurement}

\subsubsection{Demographic Variables}

Age, sex, educational level, and marital status were obtained as basic attribute items. Age was classified into two groups: "young-old" (those aged $\leq 74$ years) and "old-old" (those aged $\geq 75$ years). Participants were asked to specify their level of education (elementary school/junior high school, senior high school, technical college/junior college/vocational school, university/graduate school, and others) and classified into two groups: "elementary school to senior high school" and "higher than senior high school." Responses to the item investigating marital status (with spouse, divorced/separated, unmarried, and others) were classified into two groups: "with spouse" and "without spouse".

\subsubsection{Health Status}

Items investigating health status included body height and weight, and current disease history. Body height and weight were self-reported. Medical treatment history was determined with the question, "Do you currently receive medical treatment?" Responses of "No" were classified as "without treatment" and "Yes" as "with treatment". BMI was calculated from body height and weight, and was 
classified into three groups: "underweight $\left(<18.50 \mathrm{~kg} / \mathrm{m}^{2}\right)$ ", "normal range $(18.50$ $\left.\mathrm{kg} / \mathrm{m}^{2}-24.99 \mathrm{~kg} / \mathrm{m}^{2}\right)$ ", "overweight $\left(\geq 25 \mathrm{~kg} / \mathrm{m}^{2}\right)$ " based on the International Classification of adult underweight, overweight and obesity according to BMI [17].

\subsubsection{Physical Activity and Sitting Time}

A short Japanese version of the International Physical Activity Questionnaire was used to estimate the amount of physical activity. We calculated total physical activity time per week. The reliability and validity of this questionnaire has been reported in a previous study [18]. Based on the World Health Organization's Global Recommendations on Physical Activity for Health, physical activity time was classified as "sufficient" ( $\geq 150$ minutes of walking/moderate intensity physical activity per week), and "insufficient" ( $<150$ minutes of walking/moderate intensity physical activity per week) [19].

Sitting time was investigated across five domains: reading time, conversation with family members, use of computers, watching TV, and other lying time. The total time for each domain was calculated. Because there is no established cutoff value for sitting time for older adults, we classified sitting time as "short-time group" and "long-time group" based on the median (190 minutes).

\subsubsection{Nutritional, Smoking, and Drinking Status}

Nutritional status was determined with the question, "Have you lost $2-5 \mathrm{~kg}$ or more in the past 6 months?" Responses of "No" were classified as "Good" and "Yes" as "Poor". Smoking status was assessed with the question, "Do you smoke?" Those who indicated they currently smoked were classified as "smoker" and the remaining responses (have never smoked, smoked in the past, but do not currently smoke) as "nonsmoker." The question "Do you drink alcohol?" was used to assess drinking status, and classified as: "nondrinker" (do not drink alcohol and stopped drinking) and "drinker" (sometimes drink and drink almost every day).

\subsubsection{Knee Pain}

In this study, knee pain was assessed by "frequent knee pain", which is commonly used in epidemiological studies [20] [21]. Frequent knee pain was defined as pain in one/both knees on most days during a particular period. The definition of knee pain in this study was that used in a previous large scale cohort study [22]. Participants were asked, "Have you experienced pain in one/both knees on most days in the past one month?" Those who answered "No" were defined as "without knee pain", and those who answered "Yes" were defined as "with knee pain".

\subsection{Statistical Analysis}

Missing values were supplemented by the multiple imputation method. Multiple imputations were performed by chained equations under the assumption of missing at random. For the imputation of missing data, multiple regression 
analysis for continuous data and multiple logistic regression analysis for categorical data were applied using knee pain, demographic, health behavior, and health condition variables.

To investigate factors independently related to knee pain, multivariable logistic regression analysis was conducted using the complemented data. For the analysis model, the dependent variable was knee pain and the independent variables were physical activity, sitting time, lifestyle (nutrition, drinking, and smoking), and BMI. Adjustment variables were age, sex, educational level, marital status, and current disease history. The analytical model was fitted to 50 data sets, and odds ratios (OR) and 95\% confidence intervals (95\% CI) were calculated based on Rubin's method [23]. For the sensitivity analysis, the same analysis model as the complemented data was also applied to the complete case data, which were the data from the subjects who had no missing values. All analyses were conducted using IBM SPSS 26.0 for Windows, with a significance level of less than $5 \%$.

\section{Results}

In total, 5311 older adults responded to the survey (response rate: 79.7\%). There were 3909 valid responders who answered every item (valid response rate: 58.7\%). After multiple imputations for missing data, the data from 5311 participants were analyzed. The mean age and standard deviation of the participants were $74.5 \pm 6.8$ years for men and $74.9 \pm 6.9$ years for women. Overall, 1848 participants had knee pain, giving a prevalence rate of knee pain of $34.8 \%$. Table 1 shows the physical characteristics of the participants according to the presence or absence of knee pain. The group with knee pain had a higher BMI, shorter physical activity time, and longer sitting time than in the group without knee pain. The proportions of nonsmoker and nondrinker were higher in the group with knee pain than the group without knee pain. The proportions of those with a spouse and those with good nutrition were lower in the group with knee pain than without group. In addition, the percentages of those with a high level of education and those not receiving medical treatment were higher in the group without pain than the group with pain.

Multivariable logistic regression analysis using the complemented data showed that physical activity $(P=0.001)$, BMI $(P<0.001)$, and nutritional status $(P<0.001)$ were significantly related to knee pain. For physical activity, the OR was 0.81 and the $95 \%$ CI was 0.71 - 0.92 (reference: insufficient physical activity group). Sitting time (sedentary behavior) showed a lower OR $(0.88,95 \%$ CI: 0.78 - 1.00) in the short-time group compared with the long-time group; however, the difference between the groups did not reach statistical significance. The OR for BMI were 0.60 (95\% CI: 0.51 - 0.69), 0.38 (95\% CI: $0.29-0.51$ ), with $\leq 25$ $\mathrm{kg} / \mathrm{m}^{2}$ group as reference. For nutritional status, the OR was 0.63 (95\% CI: 0.54 $0.75)$, with the malnourished group as reference. Smoking and alcohol drinking did not show significant ORs (Table 2). The analysis using complete case data showed similar results to the complemented data. 
Table 1. Characteristics of the participants in each group with or without knee pain (Results of the analysis with multiple imputations; $\mathrm{N}=5311$ ).

\begin{tabular}{|c|c|c|c|}
\hline & Total & With knee pain & Without knee pain \\
\hline Variables & $\mathrm{N}=5311$ & $\mathrm{~N}=1848$ & $\mathrm{~N}=3463$ \\
\hline \multicolumn{4}{|l|}{ Age } \\
\hline Old-old & $2481(46.7)$ & $1070(57.9)$ & $1411(40.8)$ \\
\hline Young-old & $2830(53.3)$ & $779(42.1)$ & $2051(59.2)$ \\
\hline \multicolumn{4}{|l|}{ Sex } \\
\hline Men & 2415 (45.5) & $699(37.8)$ & $1716(49.5)$ \\
\hline Women & $2830(53.3)$ & $1149(62.2)$ & $1747(50.5)$ \\
\hline \multicolumn{4}{|l|}{ BMI } \\
\hline$\geq 25.0$ & $1178(22.3)$ & $528(28.7)$ & $650(18.8)$ \\
\hline $18.5-24.0$ & $3779(71.4)$ & $1223(66.4)$ & $2556(74.0)$ \\
\hline$<18.5$ & $338(6.4)$ & $90(4.9)$ & $248(7.2)$ \\
\hline \multicolumn{4}{|l|}{ Physical activity } \\
\hline Insufficient & $2583(48.6)$ & $1006(54.4)$ & $1578(45.6)$ \\
\hline Sufficient & $2728(51.4)$ & $842(45.6)$ & $1855(54.4)$ \\
\hline \multicolumn{4}{|l|}{ Sitting time } \\
\hline Long-time & $2723(51.3)$ & $987(53.4)$ & $1736(50.1)$ \\
\hline Short-time & $2588(48.7)$ & $861(46.6)$ & $1727(49.9)$ \\
\hline \multicolumn{4}{|l|}{ Smoking status } \\
\hline Smoker & $570(10.7)$ & $149(8.1)$ & $421(12.2)$ \\
\hline Nonsmoker & $4741(89.3)$ & 1699 (91.9) & $3041(87.8)$ \\
\hline \multicolumn{4}{|l|}{ Drinking status } \\
\hline Drinker & $1780(33.5)$ & $517(28.0)$ & $1264(36.5)$ \\
\hline Nondrinker & $3531(66.5)$ & $1332(72.0)$ & $2199(63.5)$ \\
\hline \multicolumn{4}{|l|}{ Nutritional status } \\
\hline Poor & $854(16.1)$ & $381(20.6)$ & $474(13.7)$ \\
\hline Good & 4457 (83.9) & $1468(79.4)$ & $2989(86.3)$ \\
\hline \multicolumn{4}{|l|}{ Marital status } \\
\hline Without spouse & $1588(29.9)$ & $640(34.6)$ & $948(27.4)$ \\
\hline With spouse & $3723(70.1)$ & $1208(65.4)$ & $2515(72.6)$ \\
\hline \multicolumn{4}{|l|}{ Educational level } \\
\hline Low & $4579(86.2)$ & $1649(89.2)$ & $2930(84.6)$ \\
\hline High & $732(13.8)$ & $199(10.8)$ & $533(15.4)$ \\
\hline \multicolumn{4}{|c|}{ Medical treatment history } \\
\hline Yes & $3493(65.8)$ & $1356(20.6)$ & $2136(13.7)$ \\
\hline No & $1818(34.2)$ & $492(79.4)$ & $1326(86.3)$ \\
\hline
\end{tabular}

Abbreviation: $\mathrm{BMI}=$ body mass index. Note: number of people (\%). 
Table 2. Results of logistic regression analysis for factors related to knee pain (results of the analysis with multiple imputations).

\begin{tabular}{|c|c|c|c|c|}
\hline \multirow[b]{2}{*}{ Items } & \multicolumn{4}{|c|}{$95 \%$ confidence interval } \\
\hline & Odds ratio & Lower limit & Upper limit & $P$-value \\
\hline \multicolumn{5}{|l|}{ Physical activity } \\
\hline Insufficient & 1.00 & & & \\
\hline Sufficient & 0.81 & 0.71 & 0.92 & 0.001 \\
\hline \multicolumn{5}{|l|}{ Sitting time } \\
\hline Long-time & 1.00 & & & \\
\hline Short-time & 0.88 & 0.78 & 1.00 & 0.054 \\
\hline \multicolumn{5}{|l|}{ BMI } \\
\hline$\geq 25.0$ & 1.00 & & & \\
\hline $18.5-24.0$ & 0.60 & 0.51 & 0.69 & $<0.001$ \\
\hline$<18.5$ & 0.38 & 0.29 & 0.51 & $<0.001$ \\
\hline \multicolumn{5}{|l|}{ Nutritional status } \\
\hline Poor & 1.00 & & & \\
\hline Good & 0.63 & 0.54 & 0.75 & $<0.001$ \\
\hline \multicolumn{5}{|l|}{ Smoking status } \\
\hline Smoker & 1.00 & & & \\
\hline Nonsmoker & 1.05 & 0.85 & 1.30 & 0.674 \\
\hline \multicolumn{5}{|l|}{ Drinking status } \\
\hline Drinker & 1.00 & & & \\
\hline Nondrinker & 1.06 & 0.92 & 1.22 & 0.447 \\
\hline
\end{tabular}

Abbreviation: $\mathrm{BMI}=$ body mass index. Note: Dependent variable is knee pain. Independent variables are physical activity, sitting time, lifestyle (nutrition, drinking, smoking), and BMI. Adjustment variables are age, sex, educational level, marital status, and medical treatment history.

\section{Discussion}

The strength of this study was that comprehensive factors related to knee pain were examined based on the data with low participant selection bias in the community-dwelling older adults. The results showed that one of three independent community-dwelling older adults had chronic knee pain, and that physical activity, BMI, and nutritional status were related to knee pain. These results seem to provide important information for developing measures to prevent knee pain among community-dwelling older adults.

Many previous studies reported that physical activity was related to various diseases and health problems [24]. This study provided new evidence that physical activity was related to knee pain among independent community-dwelling older adults. Previous studies also reported that physical activity level was positively correlated with muscle strength [25] [26], and that weakness of lower limb and quadriceps muscles has been related to the onset of knee OA [27], which is a 
major causal factor for knee pain. Recent systematic reviews reported that muscle strengthening and aerobic exercises were effective for improving knee pain [7] [8] [9]. These previous findings suggest that our findings regarding the association of physical activity with the prevalence of knee pain functioned through suppressing the decrease in muscular strength around the knee joint.

This study showed that both obesity (overweight) and nutritional status were related to knee pain. In this regard, a secondary relationship can be inferred through the relationship with the onset and progression of knee OA [28]. As Obesity causes excess load on the knee joint and lower limb muscle strength decreases with aging, the additional load on the knee due to obesity increases in older adults. The negative impact of obesity among older adults affects both the onset and progression of knee OA, which is the main disease underlying knee pain [28]. In terms of nutritional status, it is known that low bone density (which is the main progression factor for knee OA) occurs through decreased sex hormone (estrogen) secretion and insufficient intake of calcium and vitamin $\mathrm{D}$ [29] [30]. In this study, nutritional status was evaluated by the presence/absence of weight loss of $2-5 \mathrm{~kg}$ or more during the last six months. Such weight loss may reflect the decreased intake of calcium and vitamin D that accompany decreased calorie intake, which in turn causes low bone density.

This is the first study in the world examining the relationship between knee pain and sitting time among community-dwelling older adults. We found that there was no significant relationship between sitting time and knee pain. We found that there was no significant relationship between sitting time and knee pain. Previous studies reported that sitting time might be related to the mechanism of the lesion caused by hemodynamics in the development of diabetes or coronary artery disease [14] [15]. As the previously established relationships between sitting time (sedentary behavior) and health outcomes were independent of physical activity [14] [15], it is presumed that sitting behavior is not relevant for joint diseases caused by excess loading on joints, as found in this study. Another consideration regarding our result for sitting time is that the cutoff criterion for grouping sitting time might have influenced the results. In this study, the median value was used as the cutoff criterion because there was no standardized criterion for sitting time recommended to promote or maintain health status. Therefore, analyses using an evidence-based cutoff value for sitting time may yield different results from those found in this study.

This study had some limitations. First, although this study was a complete survey, the response rate of the questionnaire was $79.7 \%$, which means that the selection bias could not be completely excluded in this study. In general, however, a health-related questionnaire for older adults shows a poor response rate (50\% - 60\%) and more frequent missing data compared with a questionnaire for younger populations. This study had a high response rate and missing data were completed with imputation. Therefore, the effect of selection bias on the results was likely to have been minimized compared with the previous studies. Second, as this study used a cross-sectional design, the results cannot indicate cause-effect 
relationships. In particular, because the relationships examined in this study can be bidirectional, it is necessary to carefully consider the interpretation of the results. Finally, the results of this study were obtained from independently living older adults residing in a local municipality in Japan; it is unknown whether these results can be applied to older adults living in areas with different geographical, social, and cultural conditions. Although this study had the above limitations, this was the first study examining factors related to knee pain based on the data with low selection bias.

In conclusion, factors related to knee pain among community-dwelling independent older adults are low levels of physical activity in daily life, overweight/obesity, and poor nutrition. Sedentary behavior was not a relevant factor for knee pain in older adults. The findings of this study provide valuable information for developing prevention measures for knee pain in a community. A prospective cohort study is necessary to verify the risk factors for the onset of knee pain among community-dwelling independent older adults.

\section{Acknowledgements}

We would like to thank all participants who participated and cooperated with the implementation of this study, and also for Yoshikuni Fukasawa, Manager of the Longevity Care Section of the Tsuru City Welfare and Health Department, and Mutsue Amano of the Elderly Support Center (Community General Support Center), and staff in Tsuru City Hall who provided us with great support. We are grateful to Professor Susumu S Sawada for carefully proofreading the manuscript. This work was supported by a JSPS KAKENHI (Grant-in-Aid for Scientific Research (B)) Grant Number $15 \mathrm{H} 03089$ and sponsored by Japanese Physical Therapy Association (JPTA) Grant Number (29 - 310). The authors have no conflicts of interest directly relevant to the content of this article.

\section{Conflicts of Interest}

The authors declare no conflicts of interest regarding the publication of this paper.

\section{References}

[1] Gheno, R., Cepparo, J.M., Rosca, C.E. and Cotton, A. (2012) Musculoskeletal Disorders in the Elderly. Journal of Clinical Imaging Science, 2, 39. https://doi.org/10.4103/2156-7514.99151

[2] Jover, J.A., Lajas, C., Leon, L., Carmona, L., Serra, J.A., Reoyo, A., et al. (2015) Incidence of Physical Disability Related to Musculoskeletal Disorders in the Elderly: Results from a Primary Care-Based Registry. Arthritis Care \& Research, 67, 89-93. https://doi.org/10.1002/acr.22420

[3] Edwards, M.H., van der Pas, S., Denkinger, M.D., Parsons, C., Jameson, K.A., Schaap, L., et al. (2014) Relationships between Physical Performance and Knee and Hip Osteoarthritis: Findings from the European Project on Osteoarthritis (EPOSA). Age and Ageing, 43, 806-813. https://doi.org/10.1093/ageing/afu068

[4] Takemasa, S., Nakagoshi, R., Uesugi, M., Inoue, Y., Gotou, M., Koeda, H., et al. 
(2015) Factors That Affect the Quality of Life of Community-Dwelling Elderly Women with Musculoskeletal Disorders. Journal of Physical Therapy Science, 27, 3429-3431. https://doi.org/10.1589/jpts.27.3429

[5] Cross, M., Smith, E., Hoy, D., Nolte, S., Ackerman, I., Fransen, M., et al. (2014) The Global Burden of Hip and Knee Osteoarthritis: Estimates from the Global Burden of Disease 2010 Study. Annals of Rheumatic Diseases, 73, 1323-1330. https://doi.org/10.1136/annrheumdis-2013-204763

[6] Goldberg, D.S. and McGee, S.J. (2011) Pain as a Global Public Health Priority. BMC Public Health, 11, Article No.: 770. https://doi.org/10.1186/1471-2458-11-770

[7] Hochberg, M.C., Altman, R.D., April, K.T., Benkhalti, M., Guyatt, G., McGowan, J., et al. (2012) American College of Rheumatology 2012 Recommendations for the Use of Nonpharmacologic and Pharmacologic Therapies in Osteoarthritis of the Hand, Hip, and Knee. Arthritis Care \& Research (Hoboken), 64, 465-474. https://doi.org/10.1002/acr.21596

[8] Loew, L., Brosseau, L., Wells, G.A., Tugwell, P., Kenny, G.P., Reid, R., et al. (2012) Ottawa Panel Evidence-Based Clinical Practice Guidelines for Aerobic Walking Programs in the Management of Osteoarthritis. Archives of Physical Medicine and Rehabilitation, 93, 1269-1285. https://doi.org/10.1016/j.apmr.2012.01.024

[9] Zhang, W., Moskowitz, R.W., Nuki, G., Abramson, S., Altman, R.D., Arden, N., et al. (2008) OARSI Recommendations for the Management of Hip and Knee Osteoarthritis, Part II: OARSI Evidence-Based, Expert Consensus Guidelines. Osteoarthritis and Cartilage, 16, 137-162. https://doi.org/10.1016/j.joca.2007.12.013

[10] Urquhart, D.M., Tobing, J.F., Hanna, F.S., Berry, P., Wluka, A.E., Ding, C., et al. (2011) What Is the Effect of Physical Activity on the Knee Joint? A Systematic Review. Medicine \& Science in Sports \& Exercise, 43, 432-442. https://doi.org/10.1249/MSS.0b013e3181ef5bf8

[11] The American Academy of Orthopedic Surgeons Board of Directors (2013) Treatment of Osteoarthritis of the Knee. Evidence-Based Guideline, 2nd Edition. The American Academy of Orthopedic Surgeons, IL.

https://www.aaos.org/globalassets/quality-and-practice-resources/osteoarthritis-of-t he-knee/osteoarthritis-of-the-knee-2nd-editiion-clinical-practice-guideline.pdf

[12] Silverwood, V., Blagojevic-Bucknall, M., Jinks, C., Jordan, J.L., Protheroe, J. and Jordan, K.P. (2015) Current Evidence on Risk Factors for Knee Osteoarthritis in Older Adults: A Systematic Review and Meta-Analysis. Osteoarthritis Cartilage, 23, 507-515. https://doi.org/10.1016/j.joca.2014.11.019

[13] Blagojevic, M., Jinks, C., Jeffery, A. and Jordan, K.P. (2010) Risk Factors for Onset of Osteoarthritis of the Knee in Older Adults: A Systematic Review and Meta-Analysis. Osteoarthritis Cartilage, 18, 24-33. https://doi.org/10.1016/j.joca.2009.08.010

[14] Grøntved, A. and Hu, F.B. (2011) Television Viewing and Risk of Type 2 Diabetes, Cardiovascular Disease, and All-Cause Mortality: A Meta-Analysis. JAMA, 305, 2448-2455. https://doi.org/10.1001/jama.2011.812

[15] Proper, K.I., Singh, A.S., van Mechelen, W. and Chinapaw, M.J. (2011) Sedentary Behaviors and Health Outcomes among Adults: A Systematic Review of Prospective Studies. American Journal of Preventive Medicine, 40, 174-182. https://doi.org/10.1016/j.amepre.2010.10.015

[16] Eaker, S., Bergstrom, R., Bergstrom, A., Adami, H.O. and Nyren, O. (1998) Response Rate to Mailed Epidemiologic Questionnaires: A Population-Based Randomized Trial of Variations in Design and Mailing Routines. American Journal of 
Epidemiology, 147, 74-82. https://doi.org/10.1093/oxfordjournals.aje.a009370

[17] World Health Organization (2004) Global Datebase on Body Mass Index. http://www.assessmentpsychology.com/icbmi.htm

[18] Tomioka, K., Iwamoto, J., Saeki, K. and Okamoto, N. (2011) Reliability and Validity of the International Physical Activity Questionnaire (IPAQ) in Elderly Adults: The Fujiwara-Kyo Study. Journal of Epidemiology, 21, 459-465. https://doi.org/10.2188/jea.JE20110003

[19] World Health Organization (2010) Global Recommendations on Physical Activity for Health. https://www.who.int/dietphysicalactivity/global-PA-recs-2010.pdf

[20] Zhang, Y., Zhang, B., Wise, B., Niu, J. and Zhu, Y. (2009) Statistical Approaches to Evaluating the Effect of Risk Factors on the Pain of Knee Osteoarthritis in Longitudinal Studies. Current Opinion in Rheumatology, 21, 513-519. https://doi.org/10.1097/BOR.0b013e32832ed69d

[21] Fransen, M., Agaliotis, M., Bridgett, L. and Mackey, M.G. (2011) Hip and Knee Pain: Role of Occupational Factors. Best Practice \& Research Clinical Rheumatology, 25, 81-101. https://doi.org/10.1016/j.berh.2011.01.012

[22] Felson, D.T., Naimark, A., Anderson, J., Kazis, L., Castelli, W. and Meenan, R.F. (1987) The Prevalence of Knee Osteoarthritis in the Elderly. The Framingham Osteoarthritis Study. Arthritis \& Rheumatism, 30, 914-918. https://doi.org/10.1002/art.1780300811

[23] Rubin, D.B. (1987) Multiple Imputation for Nonresponse in Surveys. Wiley, New York. https://doi.org/10.1002/9780470316696

[24] Nelson, M.E., Rejeski, W.J., Blair, S.N., Duncan, P.W., Judge, J.O., King, A.C., et al. (2007) Physical Activity and Public Health in Older Adults: Recommendation from the American College of Sports Medicine and the American Heart Association. Medicine \& Science in Sports \& Exercise, 39, 1435-1445.

https://doi.org/10.1249/mss.0b013e3180616aa2

[25] Rantanen, T., Era, P. and Heikkinen, E. (1997) Physical Activity and the Changes in Maximal Isometric Strength in Men and Women from the Age of 75 to 80 Years. Journal of the American Geriatrics Society, 45, 1439-1445. https://doi.org/10.1111/j.1532-5415.1997.tb03193.x

[26] Hansen, A.W., Beyer, N., Flensborg-Madsen, T., Gronbaek, M. and Helge, J.W. (2013) Muscle Strength and Physical Activity Are Associated with Self-Rated Health in an Adult Danish Population. Preventive Medicine, 57, 792-798. https://doi.org/10.1016/j.ypmed.2013.08.029

[27] Takagi, S., Omori, G., Endo, K., Koga, Y., Nawata, A. and Emdo, N. (2018) Quadriceps Muscle Weakness Is Related to Increased Risk of Radiographic Knee OA but Not Its Progression in Both Women and Men: The Matsudai Knee Osteoarthritis Survey. Knee Surgery, Sports Traumatology, Arthroscopy, 26, 2607-2614. https://doi.org/10.1007/s00167-017-4551-5

[28] Doherty, M. (2001) Risk Factors for Progression of Knee Osteoarthritis. The Lancet, 358, 775-776. https://doi.org/10.1016/S0140-6736(01)06006-8

[29] Zebaze, R.M.D., Ghasem-Zadeh, A., Bohte, A., Iuliano-Burns, S., Mirams, M., Price, R.I., et al. (2010) Intracortical Remodelling and Porosity in the Distal Radius and Post-Mortem Femurs of Women: A Cross-Sectional Study. The Lancet, 375, 1729-1736. https://doi.org/10.1016/S0140-6736(10)60320-0

[30] NIH Consensus Development Panel on Osteoporosis Prevention Diagnosis, and Therapy (2001) Osteoporosis Prevention, Diagnosis, and Therapy. JAMA, 285, 785-795. https://doi.org/10.1001/jama.285.6.785 